University of Wollongong

Research Online

Faculty of Engineering and Information

Faculty of Engineering and Information

Sciences - Papers: Part A

Sciences

$1-1-2014$

Compressive sensing for multipolarization through-the-wall radar imaging

Abdesselam Bouzerdoum

University of Wollongong, bouzer@uow.edu.au

Jack Yang

University of Wollongong

Fok Hing Chi Tivive

University of Wollongong, tivive@uow.edu.au

Follow this and additional works at: https://ro.uow.edu.au/eispapers

Part of the Engineering Commons, and the Science and Technology Studies Commons

Research Online is the open access institutional repository for the University of Wollongong. For further information contact the UOW Library: research-pubs@uow.edu.au 


\title{
Compressive sensing for multipolarization through-the-wall radar imaging
}

\begin{abstract}
Discrimination of targets can be improved significantly by analyzing the polarization of scattered electromagnetic waves. In radar imaging, the target image can be enhanced by combining measurements from different polarizations. In this chapter, we propose a joint image formation and fusion approach for multipolarization through-the-wall radar imaging, using compressive sensing (CS). The measurements from different polarization channels are processed jointly using the multiple measurement vector (MMV) model to produce several images of the scene, each corresponding to a polarization channel.

Furthermore, the measurement vectors are fused together to form a composite measurement vector, which yields a composite image of the scene. The advantage of fusing the measurement vectors before image formation is that the measurement noise is reduced and the target information is enhanced, which leads to a more informative composite image. The MMV model enforces the same sparsity support for all formed images by reinforcing target information across channels and attenuating noise. Experimental results are presented using simulated and real data. Analysis and comparison of experimental results demonstrate the effectiveness of the proposed through-the-wall radar imaging approach, especially in the presence of high-measurement noise.
\end{abstract}

\section{Keywords}

sensing, compressive, multipolarization, imaging, wall, radar

Disciplines

Engineering | Science and Technology Studies

\section{Publication Details}

A. Bouzerdoum, J. Yang \& F. Tivive, "Compressive Sensing for Multipolarization through-the-Wall Radar Imaging," in Compressive Sensing for Urban Radar, M. G. Amin, Ed. United States: CRC Press, 2014, pp.231-250. 


\title{
Compressive Sensing for Multipolarization through-the-Wall Radar Imaging
}

\author{
Abdesselam Bouzerdoum, Jack Yang, and Fok Hing Chi Tivive
}

\section{CONTENTS}

7.1 Introduction .............................................. 232

7.2 Through-the-Wall Radar Imaging ............................. 233

7.2.1 Delay-and-Sum Beamforming ........................... 233

7.2.2 Single-Polarization Imaging Using SMV Model ............. 235

7.2.3 Multipolarization Imaging Using SMV Model .............. 237

7.3 Multipolarization Imaging Using MMV Model ................... 238

7.3.1 MMV CS Model ..................................... 238

7.3.2 Joint Image Fusion and Formation Using MMV ............ 238

7.4 Experimental Results ...................................... 240

7.4.1 Experimental Results Using Synthetic Data ................ 241

7.4.2 Experimental Results Using Real Data .................... 243

7.5 Conclusion................................................... 246

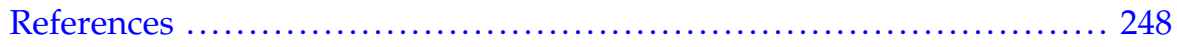

ABSTRACT Discrimination of targets can be improved significantly by analyzing the polarization of scattered electromagnetic waves. In radar imaging, the target image can be enhanced by combining measurements from different polarizations. In this chapter, we propose a joint image formation and fusion approach for multipolarization through-the-wall radar imaging, using compressive sensing (CS). The measurements from different polarization channels are processed jointly using the multiple measurement vector (MMV) model to produce several images of the scene, each corresponding to a polarization channel. Furthermore, the measurement vectors are fused together to form a composite measurement vector, which yields a composite image of the scene. The advantage of fusing the measurement vectors before image formation is that the measurement noise is reduced and the target information is enhanced, which leads to a more informative composite image. The MMV model enforces the same sparsity support for all formed images by reinforcing target information across channels and attenuating noise. Experimental results are presented using simulated and real data. 
Analysis and comparison of experimental results demonstrate the effectiveness of the proposed through-the-wall radar imaging approach, especially in the presence of high-measurement noise.

\subsection{Introduction}

Through-the-wall radar imaging (TWRI) is emerging as a viable technology to generate high-resolution images behind walls or inside enclosed building structures. TWRI systems employ electromagnetic (EM) waves that can penetrate opaque materials, such as walls and doors, to detect, recognize, and track targets inside a building. The technology has numerous civilian and military applications, for example, search-and-rescue, law enforcement, and urban surveillance and reconnaissance [3,4,6,7]. There are, however, increasing demands on TWRI systems to produce high-resolution images that can effectively discriminate the targets of interest from clutter without increasing the data acquisition and processing time.

The discrimination of the targets can be enhanced significantly by analyzing the polarization of the EM waves scattered by objects in the scene $[11,19]$. Several approaches for target detection and classification have been reported based on multiple-polarization signals. In [13], two statistical detectors were proposed for joint target detection and fusion of multipolarization radar images. In [15], a method for through-the-wall detection of small weapons was developed by exploiting target polarization signature. It was found that the ratio of the co- to cross-polarization return can be used to distinguish a human carrying a weapon from a human without a weapon. In [20], target segmentation and classification was achieved using features extracted from multipolarization images. In [26], a human detection method was proposed using a fully polarimetric scattering model of the human body.

The aforementioned studies, however, were not concerned with the problem of image formation from multiple polarizations. The problem of imaging with multiple polarizations is that the target may exhibit different responses when interrogated by different polarized signals. Therefore, the main concern of a practical full-polarization TWRI system is to effectively combine the received radar data from all polarimetric channels so as to produce an image with low background clutter and high target reflections. In [22], image fusion techniques were employed to combine the images from different polarimetric channels. In this chapter, we develop a compressed sensing (CS)-based technique for simultaneous image formation and fusion, based on multiple measurement signals.

Recently, CS has been considered for radar imaging due to its ability to reconstruct a high-resolution image from a reduced set of measurements $[2,5,17,18,23,24,31]$. The scene reconstruction is posed as an inverse problem, 
whereby a spatial map of reflections is formed from radar measurements. Most CS-based methods for TWRI exploit sparsity in a single polarimetric channel only or assume the targets have invariant reflections at different polarizations; in other words, the interchannel correlations are not fully exploited by the image formation process.

In this chapter, we present a CS approach based on the multiple measurement vectors (MMV) model, where multiple measurements from several polarizations are combined in a CS framework to compute a sparse representation of the scene. Preliminary results were presented in [29]. The scene reconstruction using multiple polarization channels is formulated as finding a sparse matrix that satisfies the measurement constraints. Compared with existing CS-based methods, the proposed approach performs simultaneous image formation and fusion, and enforces the same sparsity support across all channels. Experimental results on synthetic and real data, acquired with a stepped frequency radar, are presented, which demonstrate that the proposed method improves image quality by enhancing target reflections and attenuating background clutter.

The remainder of the chapter is organized as follows. Section 7.2 reviews the existing image formation techniques for TWRI, including delay-andsum beamforming and compressed sensing. An extension of the singlechannel CS model to multiple polarizations is also presented in this section. Section 7.3 describes the proposed MMV-based image formation approach. Section 7.4 presents experimental results, which illustrate the effectiveness of the proposed method. Finally, Section 7.5 concludes the chapter.

\subsection{Through-the-Wall Radar Imaging}

To obtain high-resolution images that can reveal objects inside an enclosed building, a ground-based TWR with a long array aperture and large bandwidth is required. The array aperture can be physical or synthesized by moving a transceiver parallel to the front wall. The data received at all antenna locations are then collected and processed to form the image of the scene behind the wall. Here, we assume the front wall reflections are removed prior to image formation $[27,28]$. Before discussing compressed sensing for TWRI, we first present a brief review of image formation using the traditional delay-and-sum (DS) beamforming in the next section.

\subsubsection{Delay-and-Sum Beamforming}

Consider a monostatic stepped-frequency TWR system with a synthetic array aperture containing $M$ antennas [4]. At each antenna location, the transceiver transmits and receives $N$ monochromatic signals of frequencies: 


$$
f_{n}=f_{1}+(n-1) \Delta f, \quad(n=1, \ldots, N)
$$

where

$f_{1}$ is the initial frequency

$\Delta f$ is the frequency step size

The transceiver is moved horizontally parallel to the wall to synthesize an array aperture. Given $P$ targets in the scene, the monochromatic signal of frequency $f_{n}$ received at the $m$ th antenna location is given by

$$
z_{m n}=\sum_{p=1}^{P} \sigma_{p} \exp \left(-j 2 \pi f_{n} \tau_{m p}\right)
$$

where

$\sigma_{p}$ is the complex reflectivity of the $p$ th target

$\tau_{m p}$ is the round-trip signal propagation delay from the $m$ th antenna location to the $p$ th target

The delay $\tau_{m p}$ is given by

$$
\tau_{m p}=\frac{2\left(d_{a}+\sqrt{\epsilon_{w}} d_{w}\right)}{c}
$$

where

$d_{a}$ is the distance traveled through the air

$\epsilon_{w}$ is the dielectric constant of the wall

$d_{w}$ is the distance traveled through the wall

$c$ is the speed of light in the air

Assume that the scene behind the wall is represented as a rectangular grid comprising $N_{x}$ and $N_{y}$ pixels along the crossrange and downrange directions, respectively. Let $\mathbf{x}$ denote the one-dimensional vector containing the image pixels arranged in a lexicographical ordering:

$$
\mathbf{x}=\left[x_{1}, \ldots, x_{q}, \ldots, x_{Q}\right]^{T}
$$

where $Q=N_{x} N_{y}$. The complex amplitude of the $q$ th pixel can be obtained by summing the delayed monochromatic signals received at all $M$ antenna locations and $N$ frequencies:

$$
x_{q}=\frac{1}{M N} \sum_{m=1}^{M} \sum_{n=1}^{N} z_{m n} \exp \left(j 2 \pi f_{n} \tau_{m q}\right),
$$


where $\tau_{m q}$ is the focusing delay between the $m$ th antenna location and the $q$ th pixel. DS beamforming utilizes all measurements to compute the complex amplitude of every pixel, thereby increasing the requirements for data acquisition and computation cost. To alleviate this problem, CS-based image formation methods have been developed for TWRI, where a reduced set of measurements is usually sufficient to recover a sparse scene. In the following section, the reconstruction of a TWRI scene from a single polarization is formulated as a single measurement vector (SMV) CS model.

\subsubsection{Single-Polarization Imaging Using SMV Model}

With the rapidly increasing demand on large-scale signal processing, it is not surprising to see CS emerging as one of the most important research areas in the past decade. CS has received considerable attention recently for its ability to perform data acquisition and compression simultaneously $[1,9,16,21]$. It can be used to reconstruct an approximation of a sparse or compressible signal from far fewer measurements than required by the sampling theorem. In TWRI, this has the advantage of reducing the number of measurement samples and data acquisition and processing time. A number of CS-based methods were proposed for TWRI in recent years $[5,8,23,24,30,31]$. In this section, we review briefly the CS-based approach for solving the image formation problem as an inverse problem using the single measurement vector model.

Suppose the received monochromatic signals are arranged into a column vector $\mathbf{z}$ of length $M N$,

$$
\mathbf{z}=\left[z_{11}, z_{21}, \ldots, z_{m n}, \ldots, z_{M N}\right]^{T},
$$

where $z_{m n}$ is the signal received by the $m$ th antenna at the $n$th frequency, see Equation 7.2. Then, Equation 7.2 can be expressed in matrix-vector form as

$$
\mathbf{z}=\Psi \mathbf{x}
$$

where $\Psi=\left[\psi_{i j}\right]$ is the so-called sensing or steering matrix. The element $\psi_{i j}$ is given by

$$
\psi_{i j}=\exp \left(-j 2 \pi f_{n} \tau_{m j}\right)
$$

where

$m=i \bmod M$

$n=1+(i-m) / M$

$\tau_{m j}$ is the round-trip propagation delay between the $m$ th antenna location and the $j$ th pixel 
In the absence of measurement noise and clutter, the $q$ th pixel value is ideally given by

$$
x_{q}=\left\{\begin{array}{cc}
\sigma_{p}, & \text { if } q \text { th pixel includes } p \text { th target } \\
0, & \text { otherwise }
\end{array}\right.
$$

where $\sigma_{p}$ is the complex reflectivity of the $p$ th target; in other words, the pixel value is nonzero only if a target exists at that pixel location. In practice, this may not be the case due to shadows and ghosts formed by multipath and multiple reflections between targets. However, in TWRI the imaged scene is usually sparsely populated, and hence the number of nonzero pixels is expected to be much smaller than the image size.

Suppose the vector $\mathbf{x}$ is $K$-sparse; that is, $\mathbf{x}$ contains at most $K$ nonzero elements with $K \ll Q$. Given a linear measurement process, represented by a matrix $\Phi$ of size $R \times M N$ (where $R<Q$ ), the measurement vector $\mathbf{y}$ can be expressed as

$$
\mathbf{y}=\Phi \mathbf{z}=\Phi \Psi \mathbf{x}=\mathbf{D x},
$$

where $\mathbf{D}=\Phi \Psi$ is known as the dictionary. CS theory allows the reconstruction of a K-sparse vector $\mathbf{x}$ from the measurements $\mathbf{y}$ by solving the following problem:

$$
\min \|\mathbf{x}\|_{1} \quad \text { subject to } \mathbf{y}=\mathbf{D} \mathbf{x},
$$

where $\|.\|_{1}$ denotes the $p$-norm (with $p=1$ ). Alternatively, if the measurement vector is corrupted by noise, a sparse signal $\mathbf{x}$ can be recovered by solving

$$
\min \|\mathbf{x}\|_{1} \quad \text { subject to }\|\mathbf{y}-\mathbf{D x}\|_{2} \leq \epsilon,
$$

where $\epsilon$ is an upper bound on the noise level (see Candes and Wakin in [1]).

The problem now is how to design a measurement matrix $\Phi$ that ensures stable recovery of a $K$-sparse vector $\mathbf{x}$ from a reduced set of measurements $\mathbf{y}$. A sufficient condition for stable recovery is incoherence between the matrices $\Phi$ and $\Psi$. For an orthogonal measurement matrix $\Phi$, the signal $\mathbf{x}$ can be recovered almost perfectly provided the number of measurements $R \approx \mathcal{O}\left(K^{2} \mu(\Phi, \Psi)\right) \log (Q)$ [8], where $\mu(\Phi, \Psi)$ is the mutual coherence between $\Phi$ and $\Psi$. If $\Phi$ contains only one nonzero element in each row, then this is equivalent to selecting a subset of antennas and frequencies to perform the measurements; in this case, $\Phi$ is referred to as a selection matrix. 


\subsubsection{Multipolarization Imaging Using SMV Model}

The extension of the SMV model to multiple polarimetric channels is given in this section. Assume we have $L$ polarimetric channels. The measurement vector of each channel can be represented as

$$
\mathbf{y}_{i}=\mathbf{D}_{i} \mathbf{x}_{i}, \quad(i=1, \ldots, L),
$$

where

$\mathbf{D}_{i}=\Phi_{i} \Psi$

$\mathbf{x}_{i}$ is a column vector containing the image of the $i$ th polarimetric channel arranged in a lexicographical ordering

Here, without loss of generality, we assume all polarimetric channels have the same steering matrix $\Psi$. The SMV model given in Equation 7.12 can be applied to each polarization channel separately. Alternatively, the problem can be formulated as a single SMV model comprising all the channels. Let $\tilde{\mathbf{y}}=\left[\mathbf{y}_{1}^{T}, \ldots, \mathbf{y}_{L}^{T}\right]^{T}$ and $\tilde{\mathbf{x}}=\left[\mathbf{x}_{1}^{T}, \ldots, \mathbf{x}_{L}^{T}\right]^{T}$ denote the composite measurement and image vectors, respectively. The corresponding composite dictionary $\tilde{\mathbf{D}}$ is obtained by arranging the individual channel dictionaries $\mathbf{D}_{i}(i=1, \ldots, L)$ along the main diagonal and setting the off-diagonal elements to zero:

$$
\tilde{\mathbf{D}}=\left[\begin{array}{ccc}
\mathbf{D}_{1} & 0 & 0 \\
0 & \ddots & 0 \\
0 & 0 & \mathbf{D}_{L}
\end{array}\right]
$$

The SMV model can now be applied to recover the composite vector $\tilde{\mathbf{x}}$ by solving

$$
\min \|\tilde{\mathbf{x}}\|_{1} \quad \text { subject to }\|\tilde{\mathbf{y}}-\tilde{\mathbf{D}} \tilde{\mathbf{x}}\|_{2} \leq \epsilon .
$$

Although the extension of SMV model to multipolarization TWRI is straightforward, the drawback is that the recovered vectors $\mathbf{x}_{i}(i=1, \ldots, L)$ are not guaranteed to have the same sparse support. Furthermore, the SMV model of (7.15) does not exploit interchannel correlations. Finally, the complexity of the problem increases with increased size of the matrix $\tilde{\mathbf{D}}$, thereby requiring larger storage and more computation time to solve the CS problem (7.15). In the next section, we present a model that exploits the interchannel correlations using a joint sparse representation to enforce the same sparsity support on the recovered signals. 


\subsection{Multipolarization Imaging Using MMV Model}

In this section, we present an MMV-based image formation method for multipolarization TWRI. First, a brief description of the multiple measurement vectors CS model is given in the next section. Then, the multipolarization TWRI problem is formulated as an inverse MMV problem.

\subsubsection{CS Model}

The MMV model processes several measurement vectors simultaneously to produce a sparse matrix solution $[10,12]$. Consider a matrix of measurements $Y \in \mathbb{C}^{R \times L}$, comprising $L$ measurement vectors, and a known dictionary $\mathbf{D}$ containing $Q$ atoms. The MMV model aims to find a sparse matrix $X$ by solving the following problem [10]:

$$
\min \mathcal{S}_{0}(X) \text { subject to } Y=\mathbf{D} X,
$$

where $\mathcal{S}_{0}(X)$ denotes the sparsity rank of the matrix $X$, which is the number of nonzero rows in $X$. In other words, the aim is to find a sparse matrix solution whose columns possess the same sparsity profile. Let $\mathbf{r}_{i}$ denote the $i$ th row of the matrix $X$. Furthermore, let us define a column vector $\mathbf{s}$ whose $i$ th element $s_{i}=\left\|\mathbf{r}_{i}\right\|_{p}$, with $p \geq 2$. The sparsity rank of the matrix $X$ is given by

$$
\mathcal{S}_{0}(X)=\|\mathbf{s}\|_{0},
$$

where $\|\cdot\|_{0}$ denotes the zero pseudo-norm or cardinality of the vector argument.

However, minimizing $\mathcal{S}_{0}(X)$ is NP-hard because an exhaustive enumeration is required in terms of all possible locations of nonzero rows in $X$. Therefore, the zero pseudo-norm is usually replaced with the one-norm, $\|\cdot\|_{1}$, resulting in the following problem:

$$
\min \mathcal{S}_{1}(X)=\|\mathbf{s}\|_{1} \quad \text { subject to } Y=\mathbf{D} X .
$$

In [10], the authors proved that minimization of $\mathcal{S}_{1}(X)$ is equivalent to minimization of $\mathcal{S}_{0}(X)$ when the sparsity rank of $X$ is sufficiently low.

\subsubsection{Joint Image Fusion and Formation Using MMV}

For a stepped-frequency TWRI system, the steering matrix $\Psi$ is related to the bandwidth of the signal and the image size, see Equation 7.8. Since the radar interrogates the same scene, all polarization channels share the same sensing 
matrix, that is, $\Psi_{i}=\Psi(i=1, \ldots, L)$. Moreover, we can choose the selection matrix to be identical for all channels, $\Phi_{i}=\Phi \forall i$. This means the same dictionary can be used across all channels, $\mathbf{D}_{i}=\mathbf{D}(i=1, \ldots, L)$. Substituting $\mathbf{D}$ for $\mathbf{D}_{i}$ in (7.13) yields the single channel measurement vector

$$
\mathbf{y}_{i}=\mathbf{D} \mathbf{x}_{i}, \quad i=1, \ldots, L .
$$

Since the vectors $\mathbf{x}_{i}(i=1, \ldots, L)$ represent images of the same scene, a final composite image of the scene can be easily obtained using image fusion techniques [22]. By contrast, here we propose first to combine the raw measurement vectors from different polarimetric channels, then perform image reconstruction based on the fused measurement vector. Specifically, a composite measurement vector $\widehat{\mathbf{y}}$ is defined as a linear combination of the measurement vectors of different polarimetric channels:

$$
\widehat{\mathbf{y}}=\sum_{i=1}^{L} w_{i} \mathbf{y}_{i}=\mathbf{D} \sum_{i=1}^{L} w_{i} \mathbf{x}_{i}=\mathbf{D} \widehat{\mathbf{x}}
$$

where $w_{i}$ s are positive weights satisfying $\sum_{i=1}^{L} w_{i}=1$. Here we employ a criterion based on mutual information (MI) to compute the weights $w_{i}$. Mutual information is used to estimate the coherence between two measurement vectors $\mathbf{y}_{i}$ and $\mathbf{y}_{j}$ :

$$
I\left(\mathbf{y}_{i}, \mathbf{y}_{j}\right)=H\left(\mathbf{y}_{i}\right)+H\left(\mathbf{y}_{j}\right)-H\left(\mathbf{y}_{i}, \mathbf{y}_{j}\right), \quad(i \neq j),
$$

where

$H\left(\mathbf{y}_{i}\right)$ is the marginal entropy

$H\left(\mathbf{y}_{i}, \mathbf{y}_{j}\right)$ is the joint entropy

The weights $w_{i}(i=1,2, \ldots, L)$ are computed as

$$
w_{i}=\frac{H\left(\mathbf{y}_{i}\right)^{2}-\sum_{j \neq i}^{L} I\left(\mathbf{y}_{j}, \mathbf{y}_{i}\right)+\sum_{j \neq i}^{L} I\left(\mathbf{y}_{j}, \mathbf{y}_{i} \mid \cup_{k \neq i, j} \mathbf{y}_{k}\right)}{H\left(\mathbf{y}_{1}, \mathbf{y}_{2}, \ldots, \mathbf{y}_{L}\right) H\left(\mathbf{y}_{i}\right)},
$$

where $I\left(\mathbf{y}_{j}, \mathbf{y}_{i} \mid \cup_{k \neq j, i} \mathbf{y}_{k}\right)$ is the conditional mutual information. The first term in the numerator of (7.22) defines the importance of the $i$ th measurement vector compared with other measurement vectors, whereas the second and third terms are used to remove the overlapping information in the first term. The rationale for computing a weighted-average measurement vector is to have a sparse solution that represents the final output image with better 
signal-to-noise ratio. It is relatively simple to show that for additive i.i.d.* measurement noise, the weighted linear combination (7.20) reduces the noise variance in $\widehat{\mathbf{y}}$, compared with the noise variance in $\mathbf{y}_{i}$.

Since the radar images the same scene, it is reasonable to assume that the images of the $L$ polarimetric channels share the same sparse support but may have different nonzero coefficients. Therefore, using an augmented measurement matrix $Y=\left[\mathbf{y}_{1}, \ldots, \mathbf{y}_{L}, \widehat{\mathbf{y}}\right]$, we can simultaneously reconstruct the vectors $\mathbf{x}_{i}$ and the composite vector $\widehat{\mathbf{x}}=\sum_{i=1}^{L} w_{i} \mathbf{x}_{i}$, using the MMV model of Equation 7.18. However, in general, the measurements are corrupted by noise; therefore, we replace the MMV problem (7.18) with the following mixed-norm regularized least-squares problem:

$$
\min \|X\|_{1,2} \text { subject to }\|Y-\mathbf{D} X\|_{F} \leq \epsilon,
$$

where

$\|\cdot\|_{1,2}$ denotes the mixed $(1,2)$-norm, which is the sum of the Euclidean norms of the rows of $X$

$\|\cdot\|_{F}$ is the Frobenius norm

$\epsilon$ is an upper bound on the noise level

The resulting solution matrix $X$ contains in its first $L$ columns the images corresponding to the individual polarimetric channels, and in the last column the image corresponding to the composite measurement vector. Consequently, this approach may be viewed as performing joint image fusion and image formation.

\subsection{Experimental Results}

In this section, the proposed MMV-based method for multipolarization TWRI is evaluated on both synthetic and real radar data. For comparison, DS beamforming and the SMV model are implemented and tested. The effectiveness of the proposed method is tested in terms of the number of selected measurements and the signal-to-noise ratio of the received signals. To evaluate different image formation algorithms, the target-to-clutter ratio (TCR) is used as a performance measure:

$$
\mathrm{TCR}=10 \log \left(\frac{1 / N_{\mathcal{B}} \sum_{q \in \mathcal{B}}\left|x_{q}\right|^{2}}{1 / N_{\mathcal{C}} \sum_{q \in \mathcal{C}}\left|x_{q}\right|^{2}}\right),
$$

* Independent and identically distributed. 
where

$x_{q}$ represents the $q$ th pixel from the image

$\mathcal{B}$ and $\mathcal{C}$ denote the target and clutter regions

$N_{\mathcal{B}}$ and $N_{\mathcal{C}}$ are the number of pixels in the regions $\mathcal{B}$ and $\mathcal{C}$, respectively

\subsubsection{Experimental Results Using Synthetic Data}

In this section, experiments are conducted using synthetic data. The simulated TWRI system is a monostatic synthetic aperture radar consisting of 71 transceivers with an aperture of $2.0 \mathrm{~m}$. The stepped-frequency signal covers a $2 \mathrm{GHz}$ bandwidth, ranging from 1 to $3 \mathrm{GHz}$, with a $5 \mathrm{MHz}$ frequency step. Thus, the radar system transmits and receives $28,471(71 \times 401)$ monochromatic signals. The imaged scene is situated behind a wall of thickness $0.15 \mathrm{~cm}$ and a dielectric constant $\epsilon_{w}=7.5$. It covers an area of $5.0 \mathrm{~m} \times 5.0 \mathrm{~m}$, that is, $5.0 \mathrm{~m}$ wide and $5.0 \mathrm{~m}$ deep. The scene is partitioned into $128 \times 128$ pixels along the crossrange and downrange directions. Three point targets are placed behind the wall at coordinates $(-1.5,4) \mathrm{m},(0,1.5) \mathrm{m}$, and $(1.5,3) \mathrm{m}$. Here, we assume the scattering matrix is symmetric, hence only three polarization channels are considered: $\mathrm{HH}, \mathrm{VV}$, and HV. Figure 7.1 illustrates the images reconstructed from the three polarimetric channels using DS
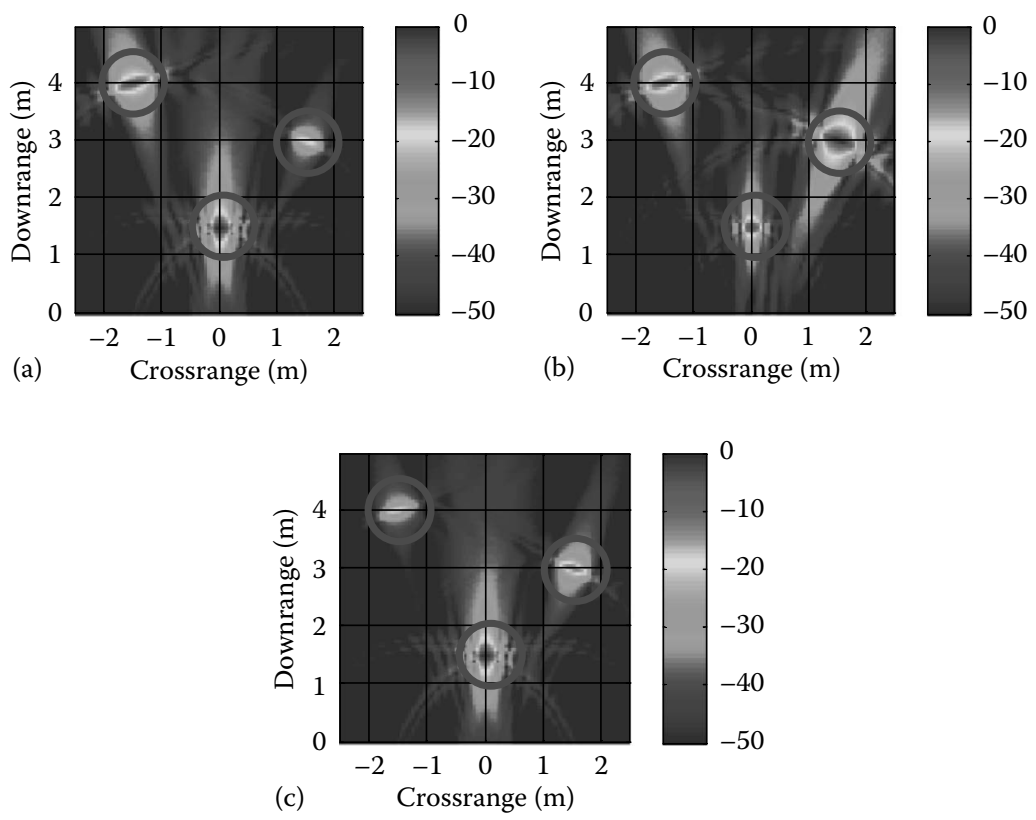

FIGURE 7.1

DS beamforming with full measurement set. The targets are simulated with different reflection coefficients at different polarization. (a) HH image, (b) HV image, and (c) VV image. 

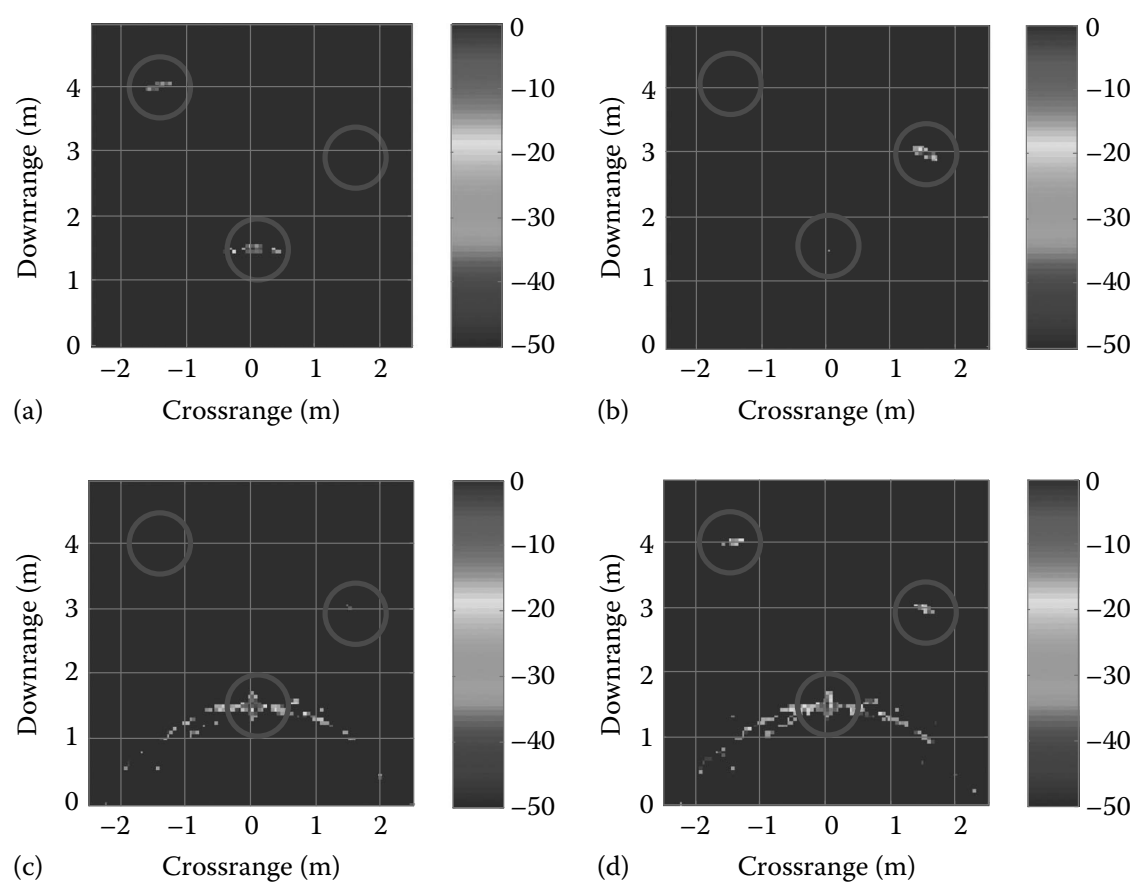

FIGURE 7.2

Images formed with 5\% measurements using SMV model of Equation 7.15. (a) HH image, (b) HV image, (c) VV image, and (d) Composite image.

beamforming with the full set of measurements. As can be observed, the formed images contain many large sidelobes around the targets.

Next, CS-based algorithms are employed to reconstruct the image of the scene using $5 \%$ of the full measurement set. Figure 7.2 illustrates the images formed by the SMV model given in Equation 7.15; the image displayed in Figure $7.2 \mathrm{~d}$ is obtained from the composite measurement vector of the three channels, given by Equation 7.20. Clearly, the images in Figure 7.2 do not share the same support, and some images fail to detect all targets. One possible reason is that the SMV model does not exploit interchannel correlations and does not impose the same sparsity profile on the reconstructed images. By contrast, Figure 7.3 depicts the images formed by the proposed MMV model given in Equation 7.23. These images share the same support, the targets are clearly visible, and the clutter is significantly reduced.

To further assess the effectiveness of the CS-based methods, the MMV and SMV models are tested on measurements corrupted by noise. Various scenarios are considered by varying the SNR and the number of measurement used to reconstruct the image of the scene. Each experiment is repeated 20 times, and the average TCR is recorded as a measure of performance. 

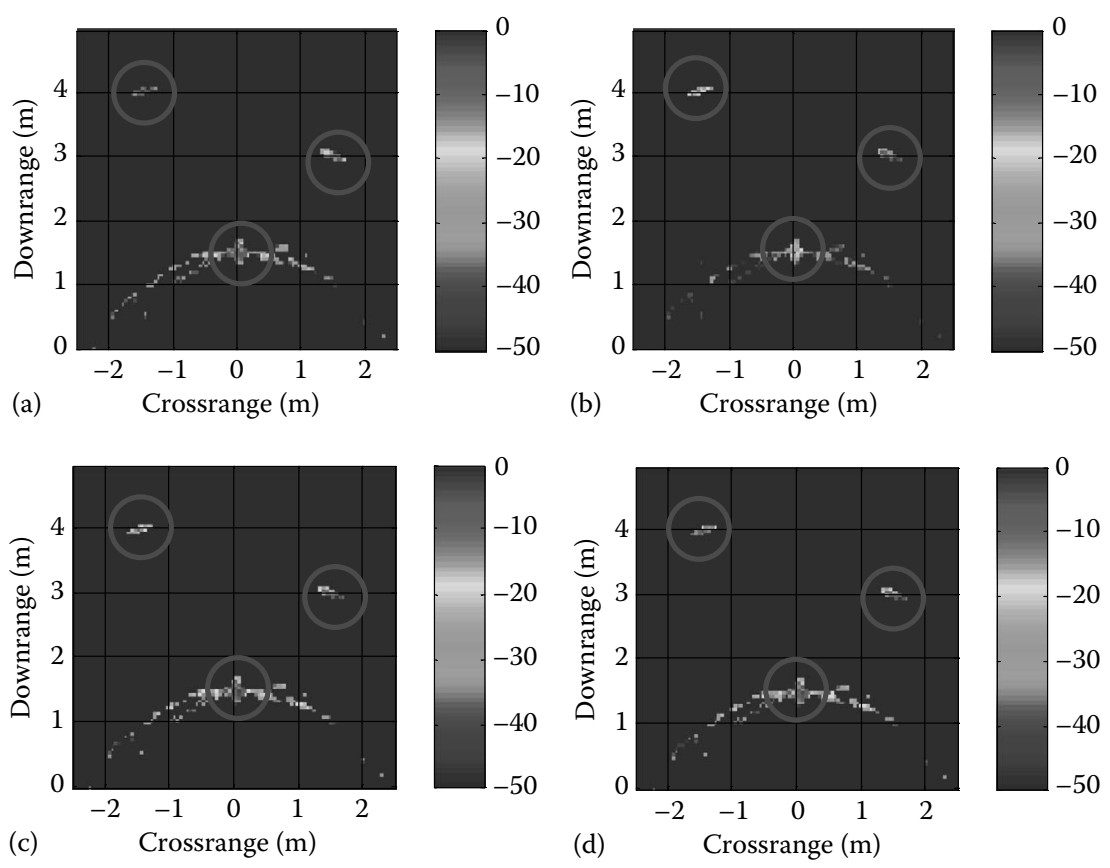

FIGURE 7.3

Images formed with 5\% measurements using MMV model of Equation 7.23. (a) HH image, (b) HV image, (c) VV image, and (d) Composite image.

The TCR is computed from the final output images obtained from the composite measurement vector $\widehat{\mathbf{y}}$ (see Figures 7.2d and 7.3d). Figure 7.4 illustrates the average TCR as a function of the number of measurements for different SNR values. As expected, increasing the percentage of measurements improves the quality of the reconstructed image for both SMV and MMV; however, MMV-based method achieves consistently higher TCR than does its SMV-based counterpart.

\subsubsection{Experimental Results Using Real Data}

Real radar data were collected at the Radar Imaging Lab of the Center for Advanced Communications, Villanova University, PA, USA. An Agilent network analyzer, model ENA-5071B with an operation frequency range of $300 \mathrm{kHz}$ to $8.5 \mathrm{GHz}$, was used to generate a stepped-frequency signal in the range $[0.7,3] \mathrm{GHz}$ with a frequency step of $2.875 \mathrm{MHz}$. A 57-element array was synthesized by mounting a horn antenna, model ETS-Lindgren $3164-04$, with an operational bandwidth of $0.7-6 \mathrm{GHz}$ on a scanner and moving the scanner horizontally at an interspacing of $0.022 \mathrm{~m}$. A room of size $7.62 \times 7.62 \mathrm{~m}$ was padded with radio frequency-absorbing material for data 

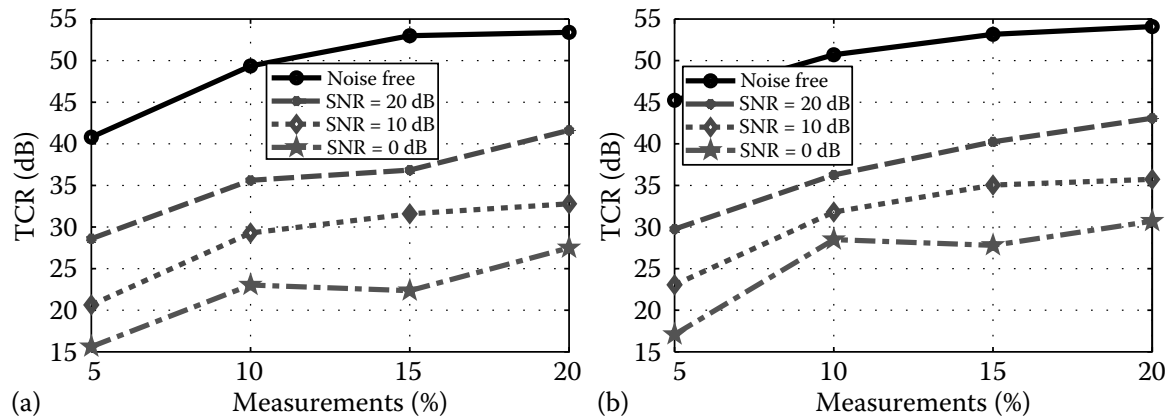

\section{FIGURE 7.4}

Average TCR as a function of number of measurements. The measurements are corrupted by additive white Gaussian noise with different SNR values. (a) SMV and (b) MMV.

collection; the interested reader is referred to [14] for more details about the room setting, the data acquisition, and the specification of the imaging system. The TWRI system was used to interrogate a scene populated with three targets placed at different locations behind a concrete wall of thickness $0.14 \mathrm{~m}$ and dielectric constant 7.6632. Figure 7.5a shows an image of the real scene with the three targets: a dihedral, a sphere, and a trihedral. Figure $7.5 \mathrm{~b}$ presents a schematic diagram of the imaged scene depicting the target locations in the downrange-crossrange plane. The total number of collected measurement samples is 45,657 (801 frequencies $\times 57$ antennas). The region of interest behind the wall is set to $4 \mathrm{~m} \times 4 \mathrm{~m}$, and the pixel size is

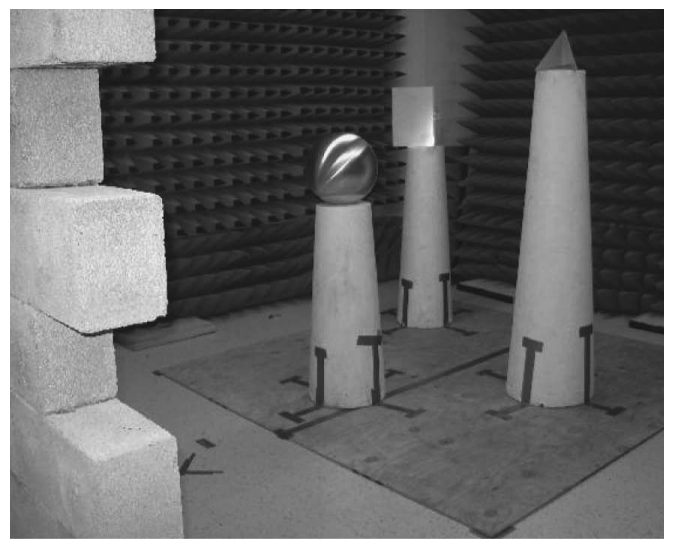

(a)

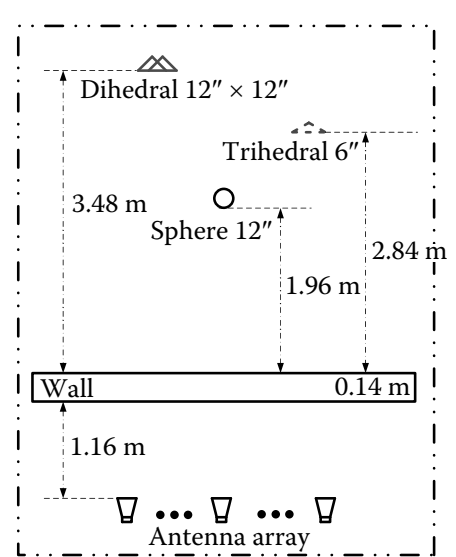

(b)

FIGURE 7.5

(a) A real scene populated with three targets: a dihedral, a sphere, and a trihedral. (b) Schematic diagram of a horizontal cross section of the imaged scene. 
set to $3.125 \mathrm{~cm} \times 3.125 \mathrm{~cm}$, resulting in an image of size $128 \times 128$ pixels. The received signals are preprocessed by removing the strong wall returns, using background subtraction, and the common signal at each antenna location. If the background signal is not available, then the strong wall returns can be mitigated using wall parameter estimation techniques that do not require the full measurement set [25].

The received signals from each polarization channel are first processed using DS beamforming to form three images. Figure 7.6 depicts the reconstructed images using the full measurement set. All images contain significant amount of clutter, but the targets are visible in the images of the copolarized channels, $\mathrm{HH}$ and VV. Figure 7.7 presents the images obtained from $15 \%$ of the measurements, selected randomly from the full measurement set. Clearly, the level of clutter increases in all images as the number of measurements is reduced.

In the remaining experiments, the CS methods are tested on reduced measurements from real data. Figure 7.8 depicts the images generated by the SMV model using $15 \%$ of the measurements. We can observe that some targets are missing from the single polarization images, and the composite image in Figure 7.8d contains a high level of clutter. In contrast, Figure 7.9 illustrates the images produced by the proposed MMV method, where all
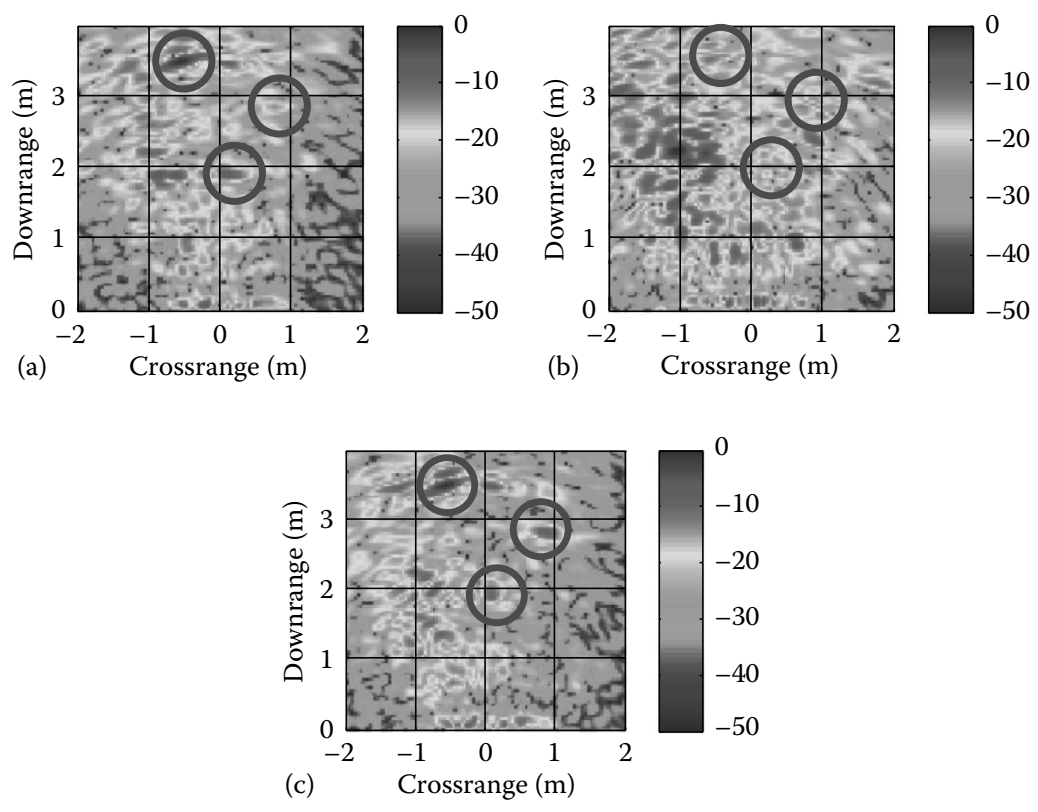

FIGURE 7.6

Reconstructed images using DS beamforming with full measurements. (a) HH image, (b) HV image, and (c) VV image. 

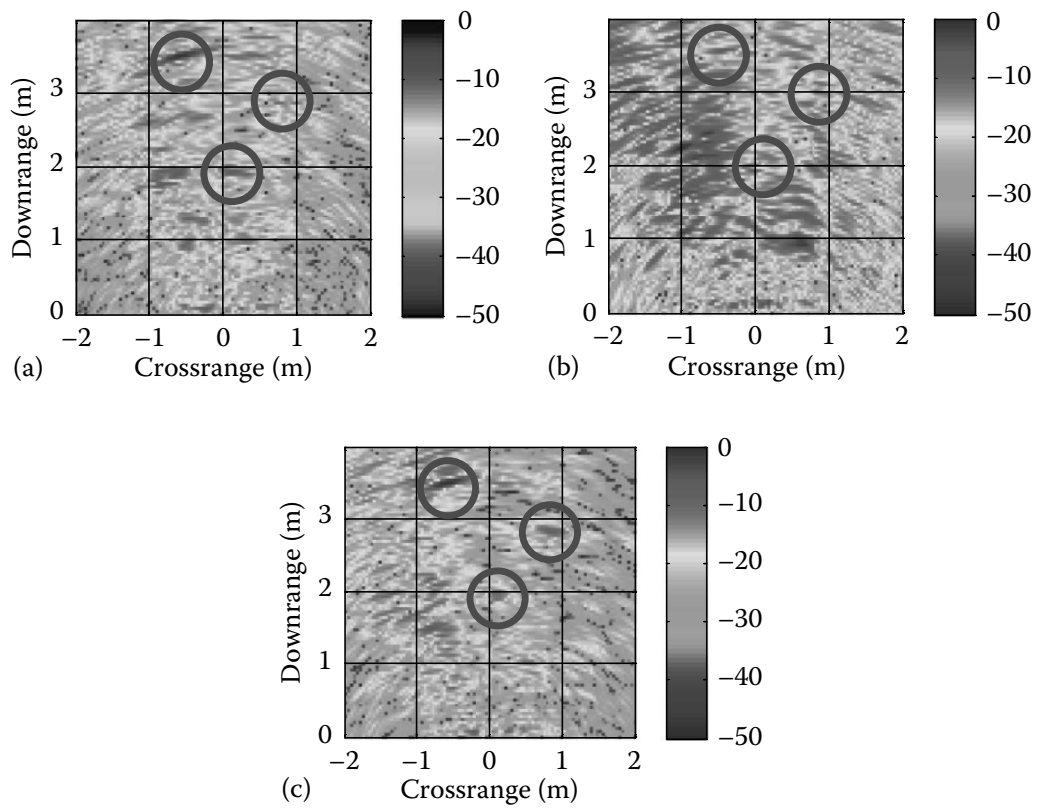

FIGURE 7.7

Reconstructed images using DS beamforming with 15\% measurements. (a) HH image, (b) HV image, and (c) VV image.

images display the targets correctly and the level of clutter is much reduced compared with the amount of clutter in the SMV generated images. Table 7.1 lists the TCR of the reconstructed composite images for different measurement percentages using DS beamforming and SMV and MMV models. Again, the quality of the reconstructed image improves as the number of measurements increases, but the MMV approach always yields a higher TCR than DS beamforming and SMV model. These experimental results confirm the superiority of the proposed image formation approach.

\subsection{Conclusion}

This chapter presented an image formation approach for multipolarization TWRI based on the multiple measurement vectors model of compressed sensing. In the MMV model, the measurement vectors obtained from different polarimetric channels are arranged into columns of a measurement matrix, which is then used to recover a sparse matrix solution whose columns constitute the images of different polarimetric channels. This is in 

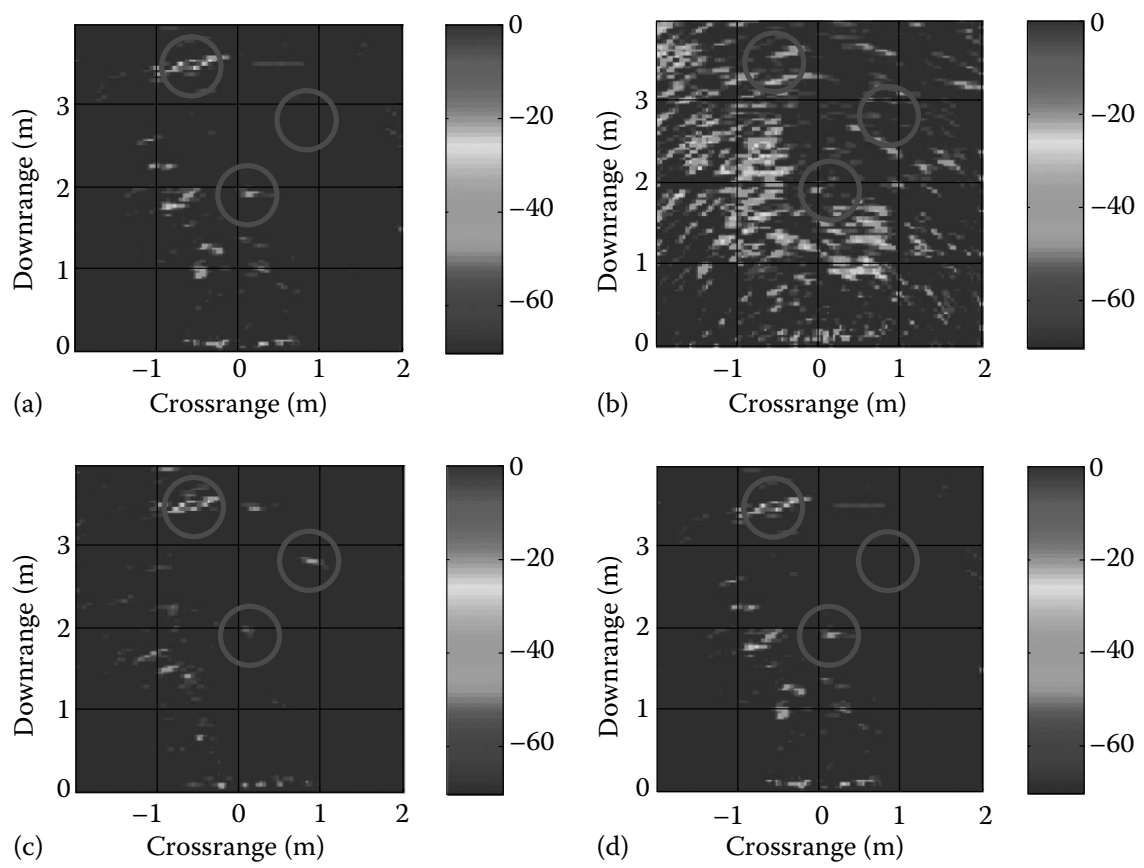

FIGURE 7.8

Reconstructed images using SMV approach with 15\% measurements. (a) HH image, (b) HV image, (c) VV image, and (d) Composite image.

contrast to the single measurement vector model, where each measurement vector is processed independently or, alternatively, all measurements are concatenated into a single measurement vector. Notwithstanding the differences in target reflectivity at different polarizations, the reconstructed channel images should ideally have the same sparsity support since they represent the same scene. The MMV model enforces the same support on all columns of the solution matrix by exploiting the interchannel correlations between the different measurement vectors. Furthermore, in the proposed approach, all channel measurement vectors are combined together to form a composite vector, which is used to reconstruct a fused image of the scene. Therefore, the proposed method can be viewed as a joint image formation and fusion approach. Experimental evaluation was conducted using synthetic and real data. The performance of the proposed approach was compared with that of the single measurement vector CS model and the traditional DS beamforming. Experimental results prove that the proposed approach reconstructs images with higher target-to-clutter ratio than does SMV or DS beamforming. In particular, the MMV images tend to have much less clutter and share the same support. 


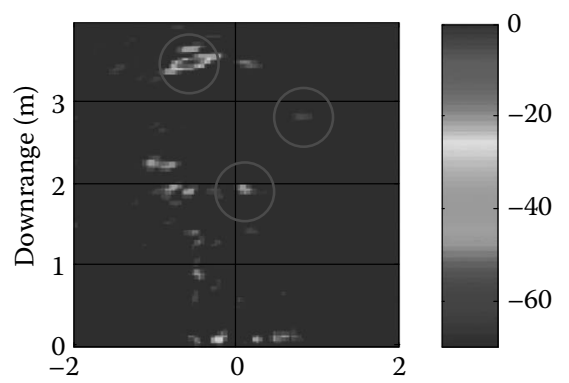

(a) Crossrange $(\mathrm{m})$

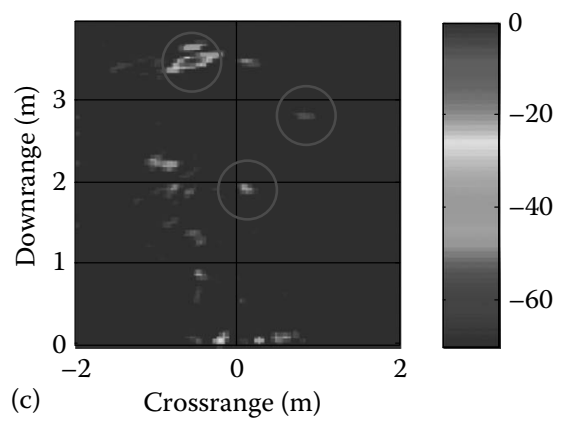

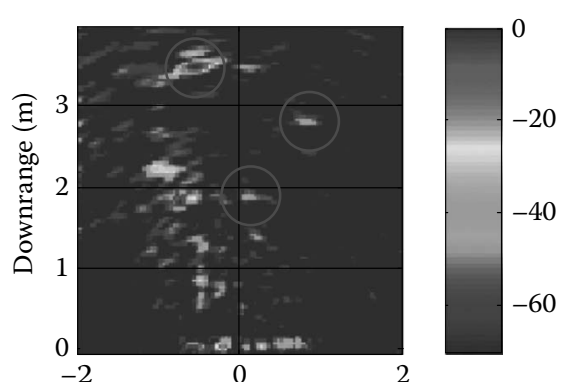

(b) Crossrange (m)

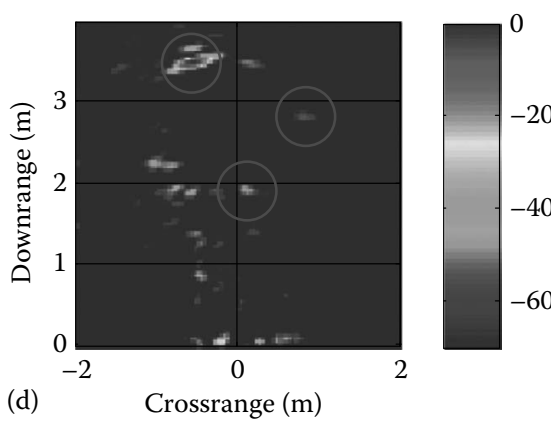

FIGURE 7.9

(See color insert.) Reconstructed images using MMV approach with $15 \%$ measurements. (a) $\mathrm{HH}$ image, (b) HV image, (c) VV image, and (d) composite image.

TABLE 7.1

TCR of Reconstructed Composite Images Using DS Beamforming, SMV, and MMV Models

\begin{tabular}{lrrr}
\hline \% Measurements & $\mathbf{5 \%}$ & $\mathbf{1 0 \%}$ & $\mathbf{1 5 \%}$ \\
\hline DS (dB) & 5.2 & 6.7 & 7.9 \\
SMV (dB) & 39.9 & 41.6 & 47.5 \\
MMV (dB) & 44.7 & 46.4 & 50.6
\end{tabular}

\section{References}

1. Special issue on sensing, sampling, and compression. IEEE Signal Processing Magazine, 25(2), March 2008.

2. E. Aguilera, M. Nannini, and A. Reigber. Multisignal compressed sensing for polarimetric SAR tomography. IEEE Geoscience and Remote Sensing Letters, 9(5):871-875, 2012. 
3. F. Ahmad and M. G. Amin. Multi-location wideband synthetic aperture imaging for urban sensing applications. Journal of the Franklin Institute, 345(6):618-639, 2008.

4. F. Ahmad, M. G. Amin, and S. A. Kassam. A beamforming approach to steppedfrequency synthetic aperture through-the-wall radar imaging. In IEEE International Workshop on Computational Advances in Multi-Sensor Adaptive Processing, Philadelphia, PA, pp. 24-27, 2005.

5. M. G. Amin and F. Ahmad. Compressive sensing for through-the-wall radar imaging. Journal of Electronic Imaging, 22(2): Article 030901, 1-21, 2013.

6. M. G. Amin and K. Sarabandi. Special issue on remote sensing of building interior. IEEE Transactions on Geoscience and Remote Sensing, 47(5):1267-1268, 2009.

7. M. G. Amin (Ed.). Through-the-Wall Radar Imaging. Boca Raton, FL: CRC Press, 2011.

8. E. Candes and J. Romberg. Sparsity and incoherence in compressive sampling. Inverse Problems, 23:969985, 2006.

9. E. Candes, J. Romberg, and T. Tao. Robust uncertainty principles: Exact signal reconstruction from highly incomplete frequency information. IEEE Transactions on Information Theory, 52:489-509, 2006.

10. J. Chen and X. Huo. Theoretical results on sparse representations of multiplemeasurement vectors. IEEE Transactions on Signal Processing, 54(12):4634-4643, 2006.

11. S. R. Cloude. Polarisation: Applications in Remote Sensing. London, U.K.: Oxford University Press, 2010.

12. S. F. Cotter, B. D. Rao, K. Engan, and K. Kreutz-Delgado. Sparse solutions to linear inverse problems with multiple measurement vectors. IEEE Transactions on Signal Processing, 53(7):2477-2488, 2005.

13. C. Debes, A. M. Zoubir, and M. G. Amin. Enhanced detection using target polarization signatures in through-the-wall radar imaging. IEEE Transactions on Geoscience and Remote Sensing, 50(5):1968-1979, 2012.

14. R. Dilsavor, W. Ailes, P. Rush, F. Ahmad, W. Keichel, G. Titi, and M. Amin. Experiments on wideband through the wall imaging. In Algorithms for Synthetic Aperture Radar Imagery XII, Vol. 5808 of Proceedings of SPIE, pp. 196-209, March 28, 2005.

15. T. Dogaru and C. Le. Through-the-wall small weapon detection based on polarimetric radar techniques. ARL-TR-5041, 2009.

16. D. Donoho. Compressed sensing. IEEE Transactions on Information Theory, 52:1289-1306, 2006.

17. A. C. Gurbuz, J. H. McClellan, and W. R. Scott. A compressive sensing data acquisition and imaging method for stepped frequency gprs. IEEE Transactions on Signal Processing, 57(7):2640-2650, 2009.

18. Q. Huang, L. Qu, B. Wu, and G. Fang. UWB through-wall imaging based on compressive sensing. IEEE Transactions on Geoscience and Remote Sensing, 48(3):1408-1415, 2010.

19. J. S. Lee and E. Pottier. Polarimetric Radar Imaging: From Basics to Applications. Boca Raton, FL: CRC Press, 2009.

20. A. A. Mostafa, C. Debes, and A. M. Zoubir. Segmentation by classification for through-the-wall radar imaging using polarization signatures. IEEE Transactions on Geoscience and Remote Sensing, 50(9):3425-3439, 2012. 
21. H. Rauhut, K. Schnass, and P. Vandergheynst. Compressed sensing and redundant dictionaries. IEEE Transactions on Information Theory, 54:2210-2219, 2008.

22. C. H. Seng, A. Bouzerdoum, M. G. Amin, and S. L. Phung. Two-stage fuzzy fusion with applications to through-the-wall radar imaging. IEEE Geoscience and Remote Sensing Letters, 10(4):687-691, July 2013.

23. V. H. Tang, A. Bouzerdoum, and S. L. Phung. Enhanced through-the-wall radar imaging using Bayesian compressive sensing. In Compressive Sensing II, Vol. 8717 of Proceedings of SPIE, Article 87170I, pp. 1-12, 2013.

24. V. H. Tang, A. Bouzerdoum, and S. L. Phung. Two-stage through-the-wall radar image formation using compressive sensing. Journal of Electronic Imaging, 22(2): Article 021006, 1-10, 2013.

25. C. Thajudeen, A. Hoorfar, and W. Zhang. Estimation of frequency-dependent parameters of unknown walls for enhanced through-the-wall imaging. In Proceedings of the IEEE International Symposium on Antennas and Propagation (AP-S/URSI), Spokane, WA, pp. 3070-3073, July 2011.

26. M. Thiel and K. Sarabandi. Ultrawideband multi-static scattering analysis of human movement within buildings for the purpose of stand-off detection and localization. IEEE Transactions on Antennas and Propagation, 59(4):1261-1268, 2011.

27. F. H. C. Tivive and A. Bouzerdoum. An improved svd-based wall clutter mitigation method for through-the-wall radar imaging. In Proceedings of the 14th IEEE Workshop on Signal Processing Advances in Wireless Communications (SPAWC), Darmstadt, Germany, pp. 425-429, June 16-19, 2013.

28. F. H. C. Tivive, A. Bouzerdoum, and M. G. Amin. An svd-based approach for mitigating wall reflections in through-the-wall radar imaging. In Proceedings of the IEEE Radar Conference (RadarCon 2011), Kansas City, MO, pp. 19-524, 2011.

29. J. Yang, A. Bouzerdoum, F. H. C. Tivive, and M. G. Amin. Multiple-measurement vector model and its application to through-the-wall radar imaging. In Proceedings of the IEEE International Conference on Acoustics, Speech and Signal Processing (ICASSP), Prague, Czech Republic, pp. 2672-2675, 2011.

30. Y.-S. Yoon and M. G. Amin. Through-the-wall radar imaging using compressive sensing along temporal frequency domain. In IEEE International Conference on Acoustics Speech and Signal Processing (ICASSP), Dallas, TX, pp. 2806-2809, 2010.

31. Y.-S. Yoon and M. G. Amin. Compressed sensing technique for high-resolution radar imaging. In Signal Processing, Sensor Fusion, and Target Recognition XVII, Vol. 6968 of Proceedings of SPIE, pp. 69681A 1-10, April 3, 2008. 